\title{
At-wavelength optics characterisation via X-ray speckle- and grating-based unified modulated pattern analysis
}

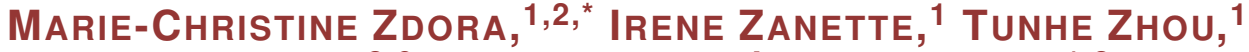 \\ FrIEDER J. KOCH, ${ }^{3,9}$ JENNY ROMELL, ${ }^{4}$ SimONE SALA, ${ }^{1,2}$ ARNDT \\ LAST, ${ }^{3}$ Yasuo OHIShI, ${ }^{5}$ NaOHISA HiRaO, ${ }^{5}$ Christoph RaU, ${ }^{1,6,7}$ \\ AND PIERRE THIBAULT ${ }^{8}$ \\ ${ }^{1}$ Diamond Light Source, Harwell Science and Innovation Campus, Didcot, Oxfordshire, OX11 ODE, UK \\ ${ }^{2}$ Department of Physics \& Astronomy, University College London, London, WC1E 6BT, UK \\ ${ }^{3}$ Institute of Microstructure Technology, Karlsruhe Institute of Technology, 76344 \\ Eggenstein-Leopoldshafen, Germany \\ ${ }^{4}$ Department of Applied Physics, Royal Institute of Technology, 10691 Stockholm, Sweden \\ ${ }^{5}$ Japan Synchrotron Radiation Research Institute (JASRI), Sayo-gun, Hyogo 679-5198, Japan \\ ${ }^{6}$ School of Materials, University of Manchester, Manchester M1 7HS, UK \\ ${ }^{7}$ Department of Otolaryngology, Northwestern University, Feinberg School of Medicine, Chicago, Illinois \\ 60611, USA \\ ${ }^{8}$ Department of Physics \& Astronomy, University of Southampton, Southampton SO17 1BJ, UK \\ ${ }^{9}$ Present address: Laboratory for Micro- and Nanotechnology, Paul Scherrer Institut, 5232 Villigen PSI, \\ Switzerland \\ *marie-christine.zdora@diamond.ac.uk
}

\begin{abstract}
The current advances in new generation X-ray sources are calling for the development and improvement of high-performance optics. Techniques for high-sensitivity phase sensing and wavefront characterisation, preferably performed at-wavelength, are increasingly required for quality control, optimisation and development of such devices. We here show that the recently proposed unified modulated pattern analysis (UMPA) can be used for these purposes. We characterised two polymer X-ray refractive lenses and quantified the effect of beam damage and shape errors on their refractive properties. Measurements were performed with two different setups for UMPA and validated with conventional X-ray grating interferometry. Due to its adaptability to different setups, the ease of implementation and cost-effectiveness, we expect UMPA to find applications for high-throughput quantitative optics characterisation and wavefront sensing.
\end{abstract}

Published by The Optical Society under the terms of the Creative Commons Attribution 4.0 License. Further distribution of this work must maintain attribution to the author(s) and the published article's title, journal citation, and DOI.

OCIS codes: (120.0120) Instrumentation, measurement, and metrology; (340.0340) X-ray optics; (110.7440) X-ray imaging; (180.7460) X-ray microscopy.

\section{References and links}

1. M. Eriksson and J. F. van der Veen, eds., Special issue on Diffraction-Limited Storage Rings and New Science Opportunities (J. Synchrotron Radiat., 2014), Vol. 21.

2. K. Ueda, ed., Special issue on X-Ray Free-Electron Laser (Appl. Sci., 2017), Vol. 7.

3. D. Malacara, ed., Optical Shop Testing (Roberts \& Company, 1992), Chap. 1.

4. P. Hariharan, "Interferometric testing of optical surfaces: absolute measurements of flatness," Opt. Eng. 36(9), 2478-2481 (1997).

5. P. Z. Takacs, S.-N. Qian, and J. Colbert, "Design of a long trace surface profiler," Proc. SPIE 0749, 59-64 (1987).

6. F. Siewert, T. Noll, T. Schlegel, T. Zeschke, and H. Lammert, "The nanometer optical component measuring machine: a new sub-nm topography measuring device for x-ray optics at BESSY," AIP Conf. Proc. 705(1), 847-850 (2004).

7. F. Siewert, J. Buchheim, T. Zeschke, M. Störmer, G. Falkenberg, and R. Sankari, "On the characterization of ultra-precise x-ray optical components: advances and challenges in ex situ metrology," J. Synchrotron Radiat. 21(5),

\#315291

Journal (C) 2018 
968-975 (2014).

8. J. Hartmann, "Bemerkungen über den Bau und die Justierung von Spektrographen," Z. Instrumentenkd. 20, 47 (1900).

9. R. V. Shack and B. C. Platt, "Production and use of a lenticular Hartmann screen," J. Opt. Soc. Am. 61, 656 (1971).

10. B. Flöter, P. Juranić, S. Kapitzki, B. Keitel, K. Mann, E. Plönjes, B. Schäfer, and K. Tiedtke, "EUV Hartmann sensor for wavefront measurements at the free-electron LASer in Hamburg," New J. Phys. 12(8), 083015 (2010).

11. B. Flöter, P. Juranić, P. Großmann, S. Kapitzki, B. Keitel, K. Mann, E. Plönjes, B. Schäfer, and K. Tiedkte, "Beam parameters of FLASH beamline BL1 from Hartmann wavefront measurements," Nucl. Instr. Meth. Phys. Res. A 635(1, Supplement), S108-S112 (2011).

12. S. C. Mayo and B. Sexton, "Refractive microlens array for wave-front analysis in the medium to hard x-ray range," Opt. Lett. 29(8), 866-868 (2004).

13. M. Idir, P. Mercere, M. H. Modi, G. Dovillaire, X. Levecq, S. Bucourt, L. Escolano, and P. Sauvageot, "X-ray active mirror coupled with a Hartmann wavefront sensor," Nucl. Instr. Meth. Phys. Res. A 616(2), 162-171 (2010).

14. C. M. Kewish, M. Guizar-Sicairos, C. Liu, J. Qian, B. Shi, C. Benson, A. M. Khounsary, J. Vila-Comamala, O. Bunk, J. R. Fienup, A. T. Macrander, and L. Assoufid, "Reconstruction of an astigmatic hard x-ray beam and alignment of K-B mirrors from ptychographic coherent diffraction data," Opt. Express 18(22), 23420-23427 (2010).

15. J. Vila-Comamala, A. Diaz, M. Guizar-Sicairos, A. Mantion, C. M. Kewish, A. Menzel, O. Bunk, and C. David, "Characterization of high-resolution diffractive x-ray optics by ptychographic coherent diffractive imaging," Opt. Express 19(22), 21333-21344 (2011).

16. T. Weitkamp, B. Nöhammer, A. Diaz, C. David, and E. Ziegler, "X-ray wavefront analysis and optics characterization with a grating interferometer," Appl. Phys. Lett. 86(5), 054101 (2005).

17. M. Engelhardt, J. Baumann, M. Schuster, C. Kottler, F. Pfeiffer, O. Bunk, and C. David, "Inspection of refractive x-ray lenses using high-resolution differential phase contrast imaging with a microfocus x-ray source," Rev. Sci. Instrum. 78(9), 093707 (2007).

18. A. Diaz, C. Mocuta, J. Stangl, M. Keplinger, T. Weitkamp, F. Pfeiffer, C. David, T. H. Metzger, and G. Bauer, "Coherence and wavefront characterization of Si-111 monochromators using double-grating interferometry," J. Synchrotron Radiat. 17(3), 299-307 (2010).

19. H. Wang, K. Sawhney, S. Berujon, E. Ziegler, S. Rutishauser, and C. David, "X-ray wavefront characterization using a rotating shearing interferometer technique," Opt. Express 19(17), 16550-16559 (2011).

20. S. Berujon and E. Ziegler, "Grating-based at-wavelength metrology of hard x-ray reflective optics," Opt. Lett. 37(21), 4464-4466 (2012).

21. Y. Kayser, C. David, U. Flechsig, J. Krempasky, V. Schlott, and R. Abela, "X-ray grating interferometer for in situ and at-wavelength wavefront metrology," J. of Synchrotron Radiat. 24(1), 150-162 (2017).

22. S. Rutishauser, I. Zanette, T. Weitkamp, T. Donath, and C. David, "At-wavelength characterization of refractive x-ray lenses using a two-dimensional grating interferometer," Appl. Phys. Lett. 99(22), 221104 (2011).

23. H. Wang, S. Berujon, and K. Sawhney, "Characterization of a one dimensional focusing compound refractive lens using the rotating shearing interferometer technique," AIP Conf. Proc. 1466(1), 223-228 (2012).

24. H. Wang, S. Berujon, and K. Sawhney, "Development of at-wavelength metrology using grating-based shearing interferometry at Diamond Light Source," J. Phys. Conf. Ser. 425(5), 052021 (2013).

25. F. J. Koch, C. Detlefs, T. J. Schröter, D. Kunka, A. Last, and J. Mohr, "Quantitative characterization of x-ray lenses from two fabrication techniques with grating interferometry," Opt. Express 24(9), 9168-9177 (2016).

26. I. Zanette, T. Weitkamp, T. Donath, S. Rutishauser, and C. David, "Two-dimensional x-ray grating interferometer," Phys. Rev. Lett. 105(24), 248102 (2010).

27. H. H. Wen, E. E. Bennett, R. Kopace, A. F. Stein, and V. Pai, "Single-shot x-ray differential phase-contrast and diffraction imaging using two-dimensional transmission gratings," Opt. Lett. 35(12), 1932-1934 (2010).

28. K. S. Morgan, P. Modregger, S. C. Irvine, S. Rutishauser, V. A. Guzenko, M. Stampanoni, and C. David, "A sensitive x-ray phase contrast technique for rapid imaging using a single phase grid analyzer," Opt. Lett. 38(22), 4605-4608 (2013).

29. K. S. Morgan, D. M. Paganin, and K. K. W. Siu, "X-ray phase imaging with a paper analyzer," Appl. Phys. Lett. 100(12), 124102 (2012).

30. S. Berujon, H. Wang, and K. Sawhney, "X-ray multimodal imaging using a random-phase object," Phys. Rev. A 86(6), 063813 (2012).

31. S. Berujon, H. Wang, and K. Sawhney, "At-wavelength metrology using the x-ray speckle tracking technique: case study of a x-ray compound refractive lens," J. Phys. Conf. Ser. 425(5), 052020 (2013).

32. S. Berujon, H. Wang, S. Alcock, and K. Sawhney, "At-wavelength metrology of hard X-ray mirror using near field speckle," Opt. Express 22(6), 6438-6446 (2014).

33. S. Berujon, E. Ziegler, and P. Cloetens, "X-ray pulse wavefront metrology using speckle tracking," J. Synchrotron Radiat. 22(4), 886-894 (2015).

34. H. Wang, Y. Kashyap, D. Laundy, and K. Sawhney, "Two-dimensional in situ metrology of X-ray mirrors using the speckle scanning technique," J. Synchrotron Radiat. 22(4), 925-929 (2015).

35. H. Wang, Y. Kashyap, and K. Sawhney, "Speckle based x-ray wavefront sensing with nanoradian angular sensitivity," Opt. Express 23(18), 23310-23317 (2015).

36. H. Wang, J. Sutter, and K. Sawhney, "Advanced in situ metrology for x-ray beam shaping with super precision," Opt. 
Express 23(2), 1605-1614 (2015).

37. Y. Kashyap, H. Wang, and K. Sawhney, "Development of a speckle-based portable device for in situ metrology of synchrotron X-ray mirrors," J. Synchrotron Radiat. 23(Pt 5), 1131-1136 (2016).

38. Y. Kashyap, H. Wang, and K. Sawhney, "Speckle-based at-wavelength metrology of x-ray mirrors with super accuracy,” Rev. Sci. Instrum. 87(5), 052001 (2016).

39. M.-C. Zdora, P. Thibault, T. Zhou, F. J. Koch, J. Romell, S. Sala, A. Last, C. Rau, and I. Zanette, "X-ray phase-contrast imaging and metrology through unified modulated pattern analysis," Phys. Rev. Lett. 118(20), 203903 (2017).

40. C. Rau, U. Wagner, Z. Pesic, and A. De Fanis, "Coherent imaging at the Diamond beamline I13," Phys. Status Solidi A 208(11), 2522-2525 (2011).

41. Federation of European Producers of Abrasives, "FEPA P-grit sizes coated abrasives," http://www . fepa-abrasives.org/Abrasiveproducts/Grains/Pgritsizescoated.aspx (accessed: 15th November 2017).

42. P. Cloetens, J. P. Guigay, C. D. Martino, J. Baruchel, and M. Schlenker, "Fractional Talbot imaging of phase gratings with hard x rays," Opt. Lett. 22(14), 1059-1061 (1997).

43. V. Nazmov, E. Reznikova, J. Mohr, A. Snigirev, I. Snigireva, S. Achenbach, and V. Saile, "Fabrication and preliminary testing of x-ray lenses in thick SU-8 resist layers," Microsys. Technol. 10(10), 716-721 (2004).

44. The refractive index decrement of SU-8 was measured at KIT/IMT in the X-ray range of $10-40 \mathrm{keV}$ and a fit to the data was performed.

45. F. Pfeiffer, M. Bech, O. Bunk, P. Kraft, E. F. Eikenberry, C. Brönnimann, C. Grünzweig, and C. David, "Hard-x-ray dark-field imaging using a grating interferometer," Nat. Mater. 7, 134-137 (2008).

46. T. Weitkamp, A. Diaz, C. David, F. Pfeiffer, M. Stampanoni, P. Cloetens, and E. Ziegler, "X-ray phase imaging with a grating interferometer," Opt. Express 13(16), 6296-6304 (2005).

47. C. Kottler, C. David, F. Pfeiffer, and O. Bunk, "A two-directional approach for grating-based differential phase contrast-imaging using hard x-rays," Opt. Express 15(3), 1175-1181 (2007).

48. B. Lengeler, C. Schroer, J. Tümmler, B. Benner, M. Richwin, A. Snigirev, I. Snigireva, and M. Drakopoulos, "Imaging by parabolic refractive lenses in the hard x-ray range," J. Synchrotron Radiat. 6(6), 1153-1167 (1999).

49. B. Lengeler, C. Schroer, B. Benner, A. Gerhardus, T. F. Günzler, M. Kuhlmann, J. Meyer, and C. Zimprich, "Parabolic refractive x-ray lenses," J. Synchrotron Radiat. 9(3), 119-124 (2002).

50. A. Snigirev, V. Kohn, I. Snigireva, and B. Lengeler, "A compound refractive lens for focusing high-energy x-rays," Nature 384, 49-51 (1996).

51. E. W. Backer, W. Ehrfeld, D. Münchmeyer, H. Betz, A. Heuberger, S. Pongratz, W. Glashauser, H. J. Michel, and R. v. Siemens, "Production of separation-nozzle systems for uranium enrichment by a combination of X-ray lithography and galvanoplastics," Naturwissenschaften 69(11), 520-523 (1982).

52. V. Saile, U. Wallradbe, O. Tabata, and J. G. Korvink, LIGA and its Applications (Wiley-VCH, 2009), Vol. 7 of Advanced Micro \& Nanosystems.

53. A. Snigirev, I. Snigireva, M. Drakopoulos, V. Nazmov, E. Reznikova, S. Kuznetsov, M. Grigoriev, J. Mohr, and V. Saile, "Focusing properties of x-ray polymer refractive lenses from SU-8 resist layer," Proc. SPIE 5195, 21-31 (2003).

54. V. Nazmov, E. Reznikova, A. Somogyi, J. Mohr, and V. Saile, "Planar sets of cross x-ray refractive lenses from SU-8 polymer," Proc. SPIE 5539, 235-243 (2004).

55. V. Nazmov, E. Reznikova, M. Boerner, J. Mohr, V. Saile, A. Snigirev, I. Snigireva, M. Di Michiel, M. Drakopoulos, R. Simon, and M. Grigoriev, "Refractive lenses fabricated by deep SR lithography and LIGA technology for X-ray energies from $1 \mathrm{keV}$ to $1 \mathrm{MeV}$," AIP Conf. Proc. 705(1), 752-755 (2004),

56. E. Reznikova, T. Weitkamp, V. Nazmov, A. Last, M. Simon, and V. Saile, "Investigation of phase contrast hard x-ray microscopy using planar sets of refractive crossed linear parabolic lenses made from SU-8 polymer," Phys. Status Solidi A 204(8), 2811-2816 (2007).

57. E. Reznikova, T. Weitkamp, V. Nazmov, M. Simon, A. Last, and V. Saile, "Transmission hard x-ray microscope with increased view field using planar refractive objectives and condensers made of SU-8 polymer," J. Phys. Conf. Ser. 186(1), 012070 (2009).

58. F. J. Koch, "X-ray optics made by x-ray lithography: Process optimization and quality control," Ph.D. thesis, Karlsruher Institut für Technologie (KIT) (2017).

59. A. Last, O. Márkus, S. Georgi, and J. Mohr, "Röntgenoptische Messung des Seitenwandwinkels direktlithografischer refraktiver Röntgenlinsen," in Proceedings of MEMS, Mikroelektronik, Systeme, Mikrosystemtechnik-Kongress 6 (VDE, 2015), pp. 508-510.

60. J. W. Goodman, Introduction to Fourier Optics (Roberts \& Company, 2004), 3rd ed.

\section{Introduction}

The current trend towards extremely brilliant and coherent X-ray beams has initiated a new era for $\mathrm{X}$-ray science, in particular in the fields of X-ray imaging and diffraction, allowing unprecedented spatial and temporal resolution [1,2]. To fully exploit the coherence properties of the X-ray beam, precise and aberration-free optical elements, such as refractive lenses, mirrors and diffractive 
optics, are desired. The need for testing and continuously improving these components has led to an increasing demand for accurate, precise and sensitive methods for optics characterisation and wavefront measurement.

Many established ex-situ metrology techniques, mostly in the visible light regime, such as the Fizeau interferometer [3,4] and slope-measuring profilers [5-7], can provide detailed and accurate information about some types of optics, e.g. mirrors, while they are less suitable for others, such as X-ray refractive lenses. Furthermore, it is important to analyse the performance of the optical elements in-situ and at-wavelength, under the same or similar conditions as they are typically used. In particular, mechanical stress, beam-induced heat load, vibrations and drifts can have an influence on the performance of the optics and should hence be comparable to the experimental conditions when performing the quality assessment.

Methods like the Hartmann [8] or Shack-Hartmann [9] sensor have been translated from the visible to the soft $[10,11]$ and hard X-ray regimes $[12,13]$. However, due to the limited spatial resolution and elaborate calibration procedure, other techniques have proven more suitable for at-wavelength metrology and wavefront measurements in the hard X-ray regime.

Ptychographic coherent diffractive imaging $[14,15]$ and X-ray grating interferometry (XGI) [16-21] are successfully being used for metrology and provide high sensitivity. Drawbacks of the techniques include the need for elaborate alignment procedures of the setup components, as well as the computational expense and vast number of frames for the former and the limited sensitivity in only one direction for the latter. Despite these limitations, XGI has become a popular method to analyse the quality of X-ray refractive lenses that are challenging to measure using ex-situ visible light methods [17,22-25]. Two-dimensional information can be achieved with grating interferometry by taking two data sets with the grating oriented in orthogonal directions [17] - at the cost of doubling the acquisition time. Alternatively, a 2D grating can be used [22,26], which is, however, very costly due to the elaborate production process.

Other approaches for 2D phase-sensitive imaging with a periodic pattern have been proposed, e.g. using an absorption grid with an analysis in Fourier space [27] or a phase grid with a correlation analysis in real space [28]. These methods allow fast single-shot acquisition, which, however, comes at the cost of reduced spatial resolution.

In the last five years, X-ray speckle-based phase-contrast imaging (SBI) $[29,30]$ has found increasing use as a cost-effective, easily implemented alternative to XGI. Compared to the other methods, SBI is more relaxed on alignment requirements and does not rely on a high degree of longitudinal nor lateral coherence of the X-ray beam. It furthermore simultaneously delivers the differential phase in two orthogonal directions. SBI has successfully been applied to wavefront measurements and metrology of X-ray refractive lenses, mirrors and Fresnel zone plates [30-38].

Recently, the unified modulated pattern analysis (UMPA) has been proposed, which overcomes some of the main drawbacks of the common implementations of XGI and SBI and allows flexible tuning of signal sensitivity and spatial resolution [39]. These properties make UMPA a suitable candidate for wavefront measurements and metrology applications with a simple setup that can be adapted to most experimental conditions. UMPA, furthermore, does not impose strong restrictions on the properties of the investigated optics as it is often the case for grating interferometry [25].

A first demonstration of optics characterisation using the UMPA method has been presented recently on a polymer X-ray refractive lens [39]. Here, we study in more detail the results of beam damage on the performance of this lens and we investigate shape errors of another undamaged polymer lens using the UMPA technique with a random as well as a periodic reference pattern.

\section{Experimental setup and data acquisition}

Measurements were conducted at the beamline I13-1 at Diamond Light Source, UK [40]. A horizontally deflecting monochromator was used to select a beam with an X-ray energy of $18.4 \mathrm{keV}$ from the undulator spectrum. The results presented here were acquired with three 


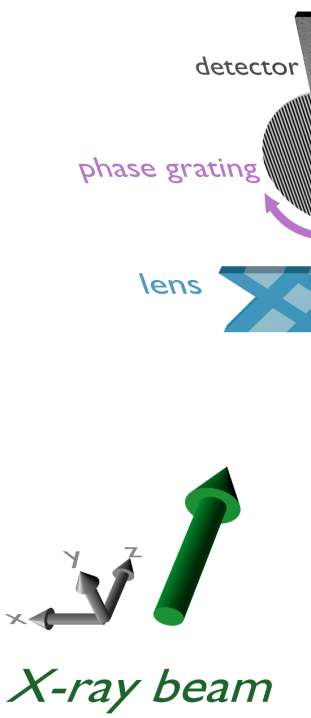

(a)

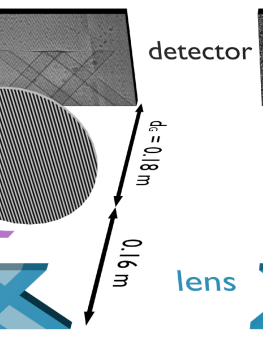

(b)

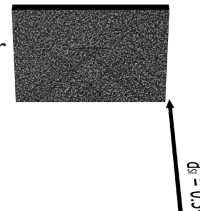

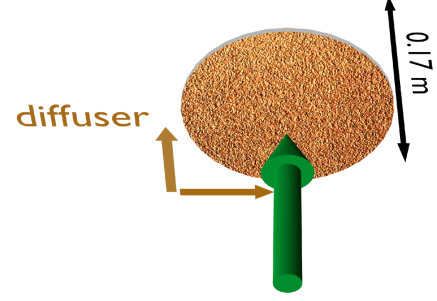

X-ray beam (c)

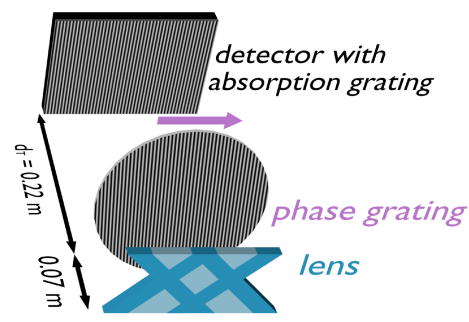

Fig. 1. Experimental arrangements for the characterisation of the refractive lenses. Setups using the UMPA method with (a) a 1D phase grating rotated around the optical axis in 46 steps and (b) a piece of sandpaper, which was translated perpendicular to the beam directions on a spiral pattern in 24 steps. (c) X-ray grating interferometry setup for validation, consisting of a 1D phase grating and a 1D absorption grating placed in front of the detector. Phase-stepping was performed with five steps over one grating period.

different setups: (a) A setup using the UMPA method with a one-dimensional (1D) beam-splitter grating (material: SU-8 photoresist polymer, period: $5.4 \mu \mathrm{m}$, line height: $26.7 \mu \mathrm{m}$, fabricated by KIT/IMT, Germany) as a phase modulator, (b) a setup using the UMPA method with a piece of sandpaper (combination of granularities P800 and P5000 [41]) as a phase modulator, and (c) for comparison a common X-ray grating interferometer consisting of a 1D phase grating (material: nickel, period: $2.4 \mu \mathrm{m}$, line height: $3 \mu \mathrm{m}$, fabricated by KIT/IMT, Germany) and a 1D absorption grating (material: gold, period: $2.4 \mu \mathrm{m}$, line height: $80 \mu \mathrm{m}$, fabricated by microworks, Germany). A schematic drawing of the three setups is shown in Fig. 1.

The sample, here a refractive lens, was located about $220 \mathrm{~m}$ downstream of the X-ray source. For the UMPA setups the detector was placed at a distance of $d_{s 1}=0.34 \mathrm{~m}$ downstream of the sample. For the first setup in Fig. 1(a) the 1D grating was placed $0.16 \mathrm{~m}$ downstream of the lens, i.e. $d_{g}=0.18 \mathrm{~m}$ upstream of the detector, while for the second setup in Fig. 1(b) the sandpaper was situated $0.17 \mathrm{~m}$ upstream of the lens. For practical reasons the lens-detector distance was kept constant for the two setups, while the order and distance between lens and phase modulator was changed. This does not affect the absolute refraction angle values, but can have an influence on the sensitivity. For the grating interferometer in Fig. 1(c) an inter-grating distance of $d_{T}=0.22 \mathrm{~m}$ was chosen, corresponding to the 5th fractional Talbot distance [42]. The refractive lens was located at a distance of $0.07 \mathrm{~m}$ upstream of the phase grating G1 (giving a lens-detector distance of $d_{s 2}=0.29 \mathrm{~m}$ ) and the absorption grating $\mathrm{G} 2$ was placed as close as possible to the front of the detector.

The detector system consisted of a scintillation screen, a 10x objective lens, a magnifying relay optics system and a pco4000 CCD camera (pixel size: $9 \mu \mathrm{m}$ ), leading to an effective pixel size of $p_{\text {eff }}=0.40 \mu \mathrm{m}$.

Data acquisition with the UMPA method was performed for the setup in Fig. 1(a) by rotating 
the grating around the beam axis from 0 to 90 degrees in increments of 2 degrees between each of the 46 acquired projections. For the setup in Fig. 1(b) the sandpaper was moved to 24 diffuser positions on a spiral pattern in the plane perpendicular to the beam direction with step sizes of about $28 \mu \mathrm{m}$. The X-ray grating interferometer in Fig. 1(c) was operated in phase-stepping mode, scanning the phase grating in the horizontal direction, perpendicular to the grating lines, in five steps over one grating period. For each of the scans, a set of reference projections without the lens in the beam and a set of sample projections with the lens in the beam were acquired. The differential phase shift signal was obtained by analysing the displacement of the interference pattern created by the phase modulator in the detector plane as described in more detail in the next section.

Two parabolic refractive lenses made from SU-8-based photo-resist polymer [43] (refractive index decrement: $\delta_{\text {SU8 }}=\left(2.7142 \times 10^{-4}\right) / E^{2}$ with $E$ being the X-ray energy in keV [44] $)$ were tested with the setups in Fig. 1: A line-focus lens (in the following referred to as lens $A$ ) with a radius of curvature $R_{A}=6.000 \mu \mathrm{m}$ and a point-focus compound refractive lens (in the following referred to as lens $B$ ) with a radius of curvature $R_{B}=19.625 \mu \mathrm{m}$, consisting of two crossed line-focussing elements each mounted with an angle of 45 degrees to the substrate surface. Lens A was intact and functional, while lens B had experienced beam damage due to intense X-ray exposure (see information in Sec. 6.2 in the Appendix).

\section{Signal reconstruction}

Both imaging methods, UMPA and classic XGI, allow the reconstruction of multimodal signals: The differential phase shift or the refraction angle in the sample, the transmission through the specimen and the dark-field signal, which is a measure of small-angle scattering in the sample $[39,45]$. For this study, we only analyse the differential phase signal as it is the relevant modality for characterising optical elements such as X-ray refractive lenses.

UMPA - as operated here - can deliver the refraction information in the horizontal as well as the vertical direction, while XGI in its most common implementation with a $1 \mathrm{D}$ grating in phase stepping mode only delivers the refraction in the direction perpendicular to the grating lines.

\subsection{Unified modulated pattern analysis}

For the measurements using the UMPA setups in Figs. 1(a) and 1(b) with the rotating grating or the sandpaper, respectively, data analysis was performed following the formalism described in detail in [39]. The reference interference pattern produced by the phase modulator (grating or sandpaper) is modulated in position, intensity and amplitude by the refraction, absorption and small-angle scattering occurring in the sample. Direct comparison of the interference patterns with and without the sample allows one to obtain the differential phase shift, transmission and dark-field signals of the specimen under investigation. The UMPA reconstruction approach uses a model of the sample interference pattern $I_{j}(m, n)$ at a diffuser position $j$ for each pixel $(m, n)$, expressed as a modulation of the corresponding reference interference pattern $I_{0 j}(m, n)$ :

$$
I_{j}(m, n)=T(m, n)\left[\langle I\rangle+D(m, n)\left(I_{0 j}\left(m+u_{x}, n+u_{y}\right)-\langle I\rangle\right)\right]
$$

where $T(m, n)$ is the transmission through the sample, $D(m, n)$ the visibility contrast or dark-field signal, related to small-angle scattering, $u_{x}, u_{y}$ the displacement of the interference pattern in the horizontal and vertical directions, respectively, and $\langle I\rangle$ the mean intensity of the reference pattern. This model assumes a rigid translation of the interference pattern and distortions of the pattern are not considered here. A least-squares minimisation between model and measured intensity with a sum over all diffuser positions is used to retrieve $u_{x}, u_{y}, T$ and $D$. This is performed pixel-wise with an analysis window of extent $w \times w$ around each pixel $(m, n)$ and the information from the $N$ different phase modulator positions is combined. The extent of the window can be 
chosen depending on the desired resolution and signal sensitivity. From the displacement $\left(u_{x}, u_{y}\right)$ of the pattern, the refraction angle $\left(\alpha_{x}, \alpha_{y}\right)$ in the sample can be determined in small-angle approximation using the relation:

$$
\left(\alpha_{x}, \alpha_{y}\right)=\left(u_{x}, u_{y}\right) \frac{p_{\text {eff }}}{d}
$$

where $d=d_{s 1}$ for the sandpaper setup and $d=d_{g}$ for the grating case. The refraction angle is furthermore directly related to the differential phase shift $(\partial \Phi / \partial x, \partial \Phi / \partial y)$ through the X-ray wavelength $\lambda$ :

$$
\left(\frac{\partial \Phi}{\partial x}, \frac{\partial \Phi}{\partial y}\right)=\frac{2 \pi}{\lambda}\left(\alpha_{x}, \alpha_{y}\right)
$$

\subsection{X-ray grating interferometry}

The grating interferometry measurements were performed with a two-grating setup in phasestepping mode and the data was analysed with the commonly used approach based on Fourier series representation [46]. From the measured data a sample phase-stepping curve $I^{s}\left(m, n ; x_{g}\right)$ with the lens, as well as a reference curve $I^{r}\left(m, n ; x_{g}\right)$ without the lens in the beam are obtained for each pixel $(m, n)$, mapping the measured intensity oscillations to the grating position $x_{g}$. These oscillations can be approximated by sinusoidal curves, equivalent to a Fourier series with only the zeroth and first order components [45]:

$$
I^{r, s}\left(m, n ; x_{g}\right)=a_{0}^{r, s}(m, n)+a_{1}^{r, s}(m, n) \cos \left(\frac{2 \pi}{p} x_{g}+\phi_{1}^{r, s}(m, n)\right),
$$

with the zeroth and first order amplitude coefficients $a_{0}^{r, s}$ and $a_{1}^{r, s}$, the phase coefficients $\phi_{1}^{r, s}$ and the period $p$ of the analyser grating. The differential phase shift $\partial \Phi / \partial x$ and refraction angle $\alpha_{x}$ in the direction perpendicular to the grating lines can be determined from the difference in the reference and sample phases $\phi_{1, s}$ and $\phi_{1, r}$, respectively:

$$
\frac{\partial \Phi}{\partial x}=\frac{2 \pi}{\lambda} \alpha_{x}=\frac{p}{\lambda d_{T}}\left(\phi_{1, s}-\phi_{1, r}\right)
$$

where $d_{T}$ is the inter-grating distance and $\lambda$ the X-ray wavelength. The transmission signal $T$ is given by the ratio of zeroth order Fourier coefficients of the sample and reference scans: $T=a_{0}^{s} / a_{0}^{r}$. The ratio $v_{r, s}=a_{1}^{r, s} / a_{0}^{r, s}$ is commonly defined as the visibility of the interference pattern. The small-angle scattering or dark-field signal $D$, which shows as a reduction in visibility $v$ of the pattern, can hence be quantified with the expression $D=v_{s} / v_{r}=a_{1}^{s} /\left(T a_{1}^{r}\right)$.

\subsection{Phase integration}

The absolute phase shift of the X-rays induced by the lens was obtained by Fourier integration of the horizontal and vertical differential phase signals [47] for the UMPA reconstructions. For the XGI case, the phase shift was determined by simple 1D integration of the differential phase signal in the horizontal direction.

\section{Lens characterisation}

State-of-the-art X-ray refractive lenses typically have a parabolic shape to achieve line- or point-focussing of the beam without spherical aberrations [48,49]. The refraction angle $\alpha$ in the lens is then a linear function along the lens profile in the focussing direction and the focal length $f$ is directly related to the refraction angle:

$$
\begin{aligned}
& 1 / f_{x}=\frac{\partial \alpha_{x}}{\partial x} \\
& 1 / f_{y}=\frac{\partial \alpha_{y}}{\partial y}
\end{aligned}
$$


for the focal lengths $f_{x}, f_{y}$ in the horizontal direction $x$ and vertical direction $y$, respectively. For a perfect point-focussing parabolic lens without astigmatism, $f_{x}$ and $f_{y}$ should have the same value. Furthermore, the focal length $f$ is given by the radius of curvature $R$ at the apex of the lens parabola and the refraction index decrement $\delta$ of the lens material [50]:

$$
f=\frac{R}{2 \delta} .
$$

In the last decade, SU-8 polymer lenses fabricated via deep X-ray lithography [51,52] have found increasing applications [43,53-57]. The fabrication step allows high aspect ratios and precise alignment of the lens elements. Its transparency and stability to X-ray radiation make SU-8 a suitable material for compound refractive lenses (CRLs).

Here, we look at two X-ray refractive lenses fabricated from SU-8 polymer: a line-focussing lens (lens A) that had not been subject to any external damage and a point-focussing lens (lens B) that had experienced prolonged exposure to an intense X-ray beam at SPring-8 synchrotron, see Sec. 6.2 of the appendix. Using the UMPA technique, we quantify the changes in focussing behaviour of the two lenses caused by extreme X-ray beam exposure and by shape errors and investigate their implications on the focussing properties.

\subsection{Refraction angle and wavefront}

The refraction angle signals in the horizontal (focussing) direction of the line-focus lens A are presented in Figs. 2(a) and 2(b) for the UMPA sandpaper setup and UMPA grating setup, respectively. Figure 2(c) shows the phase shift integrated from the UMPA grating differential phase results. The horizontal line profiles through the middle of the lens in Figs. 2(d) and 2(e) show a globally linear behaviour of the refraction angle along the lens aperture and a parabolic shape of the phase shift. Excellent agreement between the results of the two setups can be observed.

The structures visible on the left and right side of the focussing aperture are support elements for stabilisation of the lens.

Figure 3 shows the refraction angle signals and integrated phase shift of the inner focussing aperture of the point-focus CRL (lens B) for the different imaging setups. The refraction angles in the horizontal direction measured with the UMPA sandpaper setup, the UMPA grating setup and the XGI setup, respectively, in Figs. 3(a)-3(c) show good agreement between the different data sets, as confirmed by the line plots through the centre of the lens in Fig. 3(j). Slight deviations are due to the fact that the lens was removed from the sample stage between the scans causing small differences in its position. Furthermore, it should be noted that the propagation distance from the lens to the detector was different for the UMPA setups $\left(d_{s 1}=0.34 \mathrm{~m}\right)$ and the XGI setup $\left(d_{s 2}=0.29 \mathrm{~m}\right)$ due to practical limitations. This leads to a small difference in the demagnification of the lens aperture caused by the focussing effect of the lens, which hence appears slightly wider for the XGI case. This, however, does not affect the quantitative analysis of the refraction properties. Furthermore, it can be observed that the images obtained from the setups using gratings (Figs. 3(b)-3(d) and 3(f), 3(h)) appear more blurred than for the UMPA sandpaper setup. This is likely to be caused by the beam splitting effect of the grating, which leads to a reduced resolution when the resolving power of the detector system is better than the separation of the first order diffracted beams.

The line profiles in Figs. 3(j) and 3(k) through the refraction angle in the horizontal and vertical directions, shown in Figs. 3(a)-3(c) and Figs. 3(e)-3(f), respectively, clearly demonstrate a deviation from the expected linear focussing behaviour, in particular for the vertical case. Due to the limitation of the phase sensitivity in the horizontal direction, the strong defects in the vertical focussing direction cannot be measured using XGI in this configuration, while UMPA can deliver the information in both directions. This also leads to an improved integrated phase signal with 


\section{Optics EXPRESS}
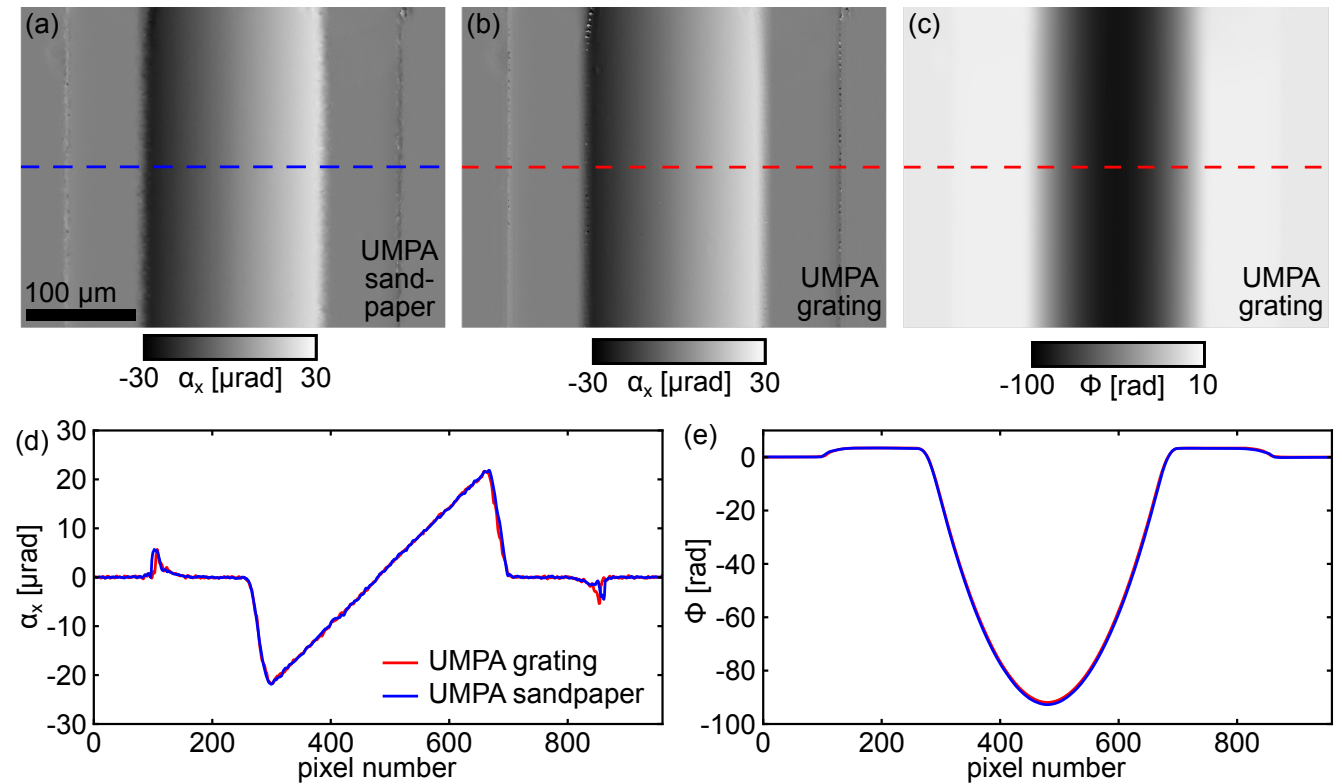

Fig. 2. Refraction angle $\alpha_{x}$ in the horizontal direction of the line-focus lens A measured with UMPA using (a) a piece of sandpaper, (b) a periodic phase grating as a phase modulator, and (c) total phase shift integrated from (b). Line profiles along the mid-line of the lens for (d) the horizontal refraction angles and (e) the integrated phase shift signals. Support elements can be observed on both sides of the lens aperture.

significantly reduced artefacts for the UMPA setups, see Figs. 3(g) and 3(h), compared to the phase signal obtained from XGI in Fig. 3(d). The parabolic shape of the wavefront after the lens is visualised in Fig. 3(i) as a surface plot of the integrated phase signal from the UMPA grating setup in Fig. 3(h).

\subsection{Lens defects and aberrations}

In the previous section, the refraction behaviour of the X-ray lenses was analysed qualitatively. While by visual inspection of the line profiles lens A showed an overall linear refraction angle along the lens profile as expected, deviations from the design refraction behaviour were observed for the damaged lens B. In this section, the effects of defects and shape errors of the lenses on the wavefront gradient are investigated in a quantitative manner.

\subsubsection{Deviations from the expected refraction behaviour}

The lenses are designed to produce a known parabolic phase shift of the X-ray beam, i.e. a linear refraction angle. A straightforward way to analyse defects and errors is to look at the deviation from this expected refraction signal, i.e. the wavefront gradient error. Here, we calculated the residuals from a linear fit to the reconstructed refraction angle, as proposed in [25]. Figures 4(a), 4(d), and 4(g) show the measured deviation in the aperture of the damaged point-focus lens B from the expected linear refraction in the horizontal direction for the UMPA sandpaper, the UMPA grating and the XGI setups, respectively. The results agree well for the different setups, confirming the validity and reproducibility of the UMPA method. The same analysis was performed for the refraction in the vertical direction for the two UMPA setups in Figs. 4(b) and 4(e), revealing even stronger aberrations from a linear behaviour. Figures 4(c), 4(f), and 4(h) display the absolute deviation, i.e. the square root of the summed squares of the contributions 


\section{Optics EXPRESS}

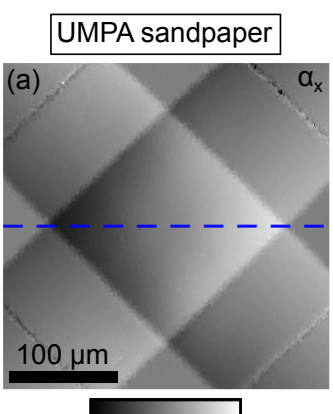

$-11 \alpha[\mu \mathrm{rad}] 11$

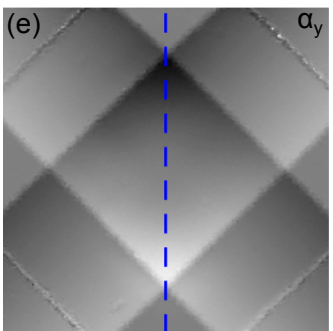

(g)

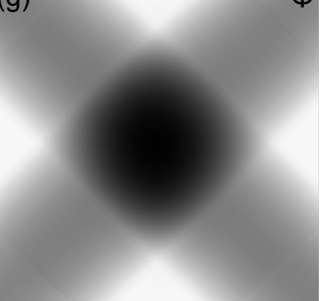

$-51 \Phi[\mathrm{rad}] 0$
UMPA grating

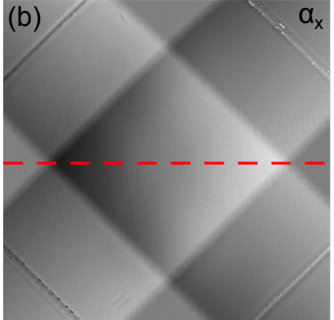

$-11 \alpha[\mu \mathrm{rad}] 11$
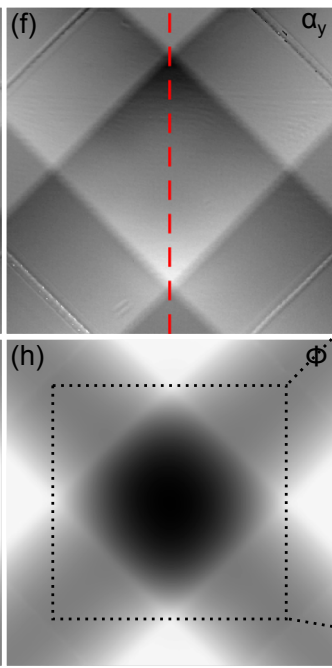

$-51 \Phi[\mathrm{rad}] \quad 0$

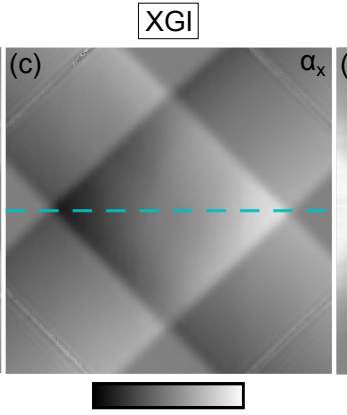

$-11 \alpha[\mu \mathrm{rad}] 11$

(i)
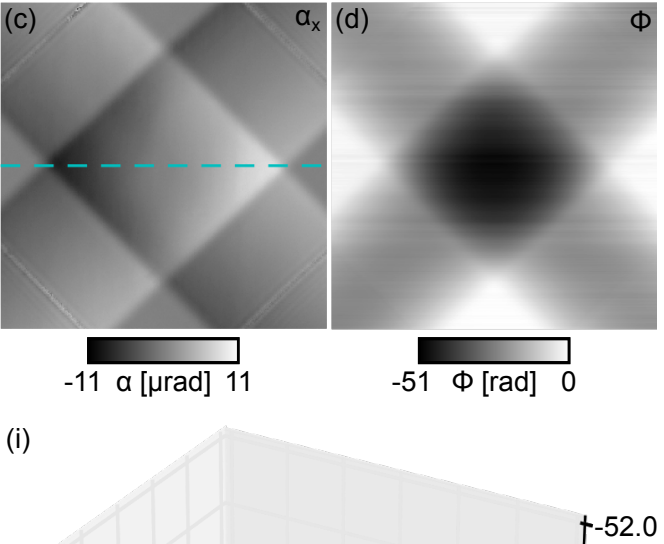

$51+[\mathrm{rad}]$
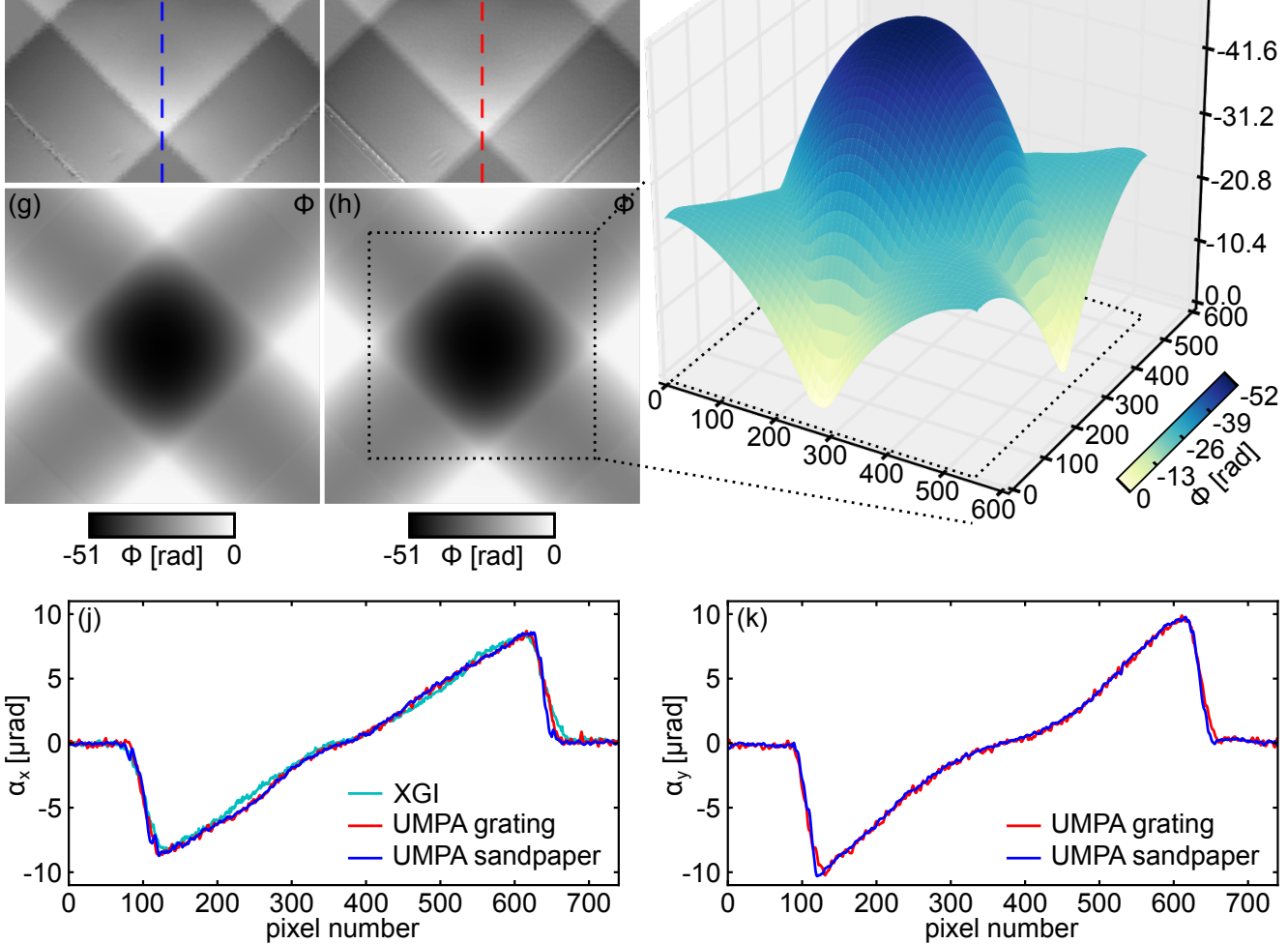

Fig. 3. Refraction angle signals $\alpha$ and integrated phase shifts $\Phi$ of the point-focus lens B. Refraction angle $\alpha_{x}$ in the horizontal direction measured with UMPA using (a) sandpaper, (b) a periodic phase grating and (c) conventional two-grating XGI. (d) Absolute phase shift determined by 1D integration of (c). Refraction angle $\alpha_{y}$ in the vertical direction measured with UMPA using (e) sandpaper, (f) a periodic phase grating and (g), (h) absolute phase shift signals integrated from (a) and (e) or (b) and (f), respectively. (i) 3D surface plot of the beam phase profile after lens B in panel (h) measured in the detector plane with the UMPA grating setup. (j), (k) Profile plots through the horizontal and vertical refraction angle signals in (a)-(c) and (e)-(f), respectively. 


\section{Optics EXPRESS}
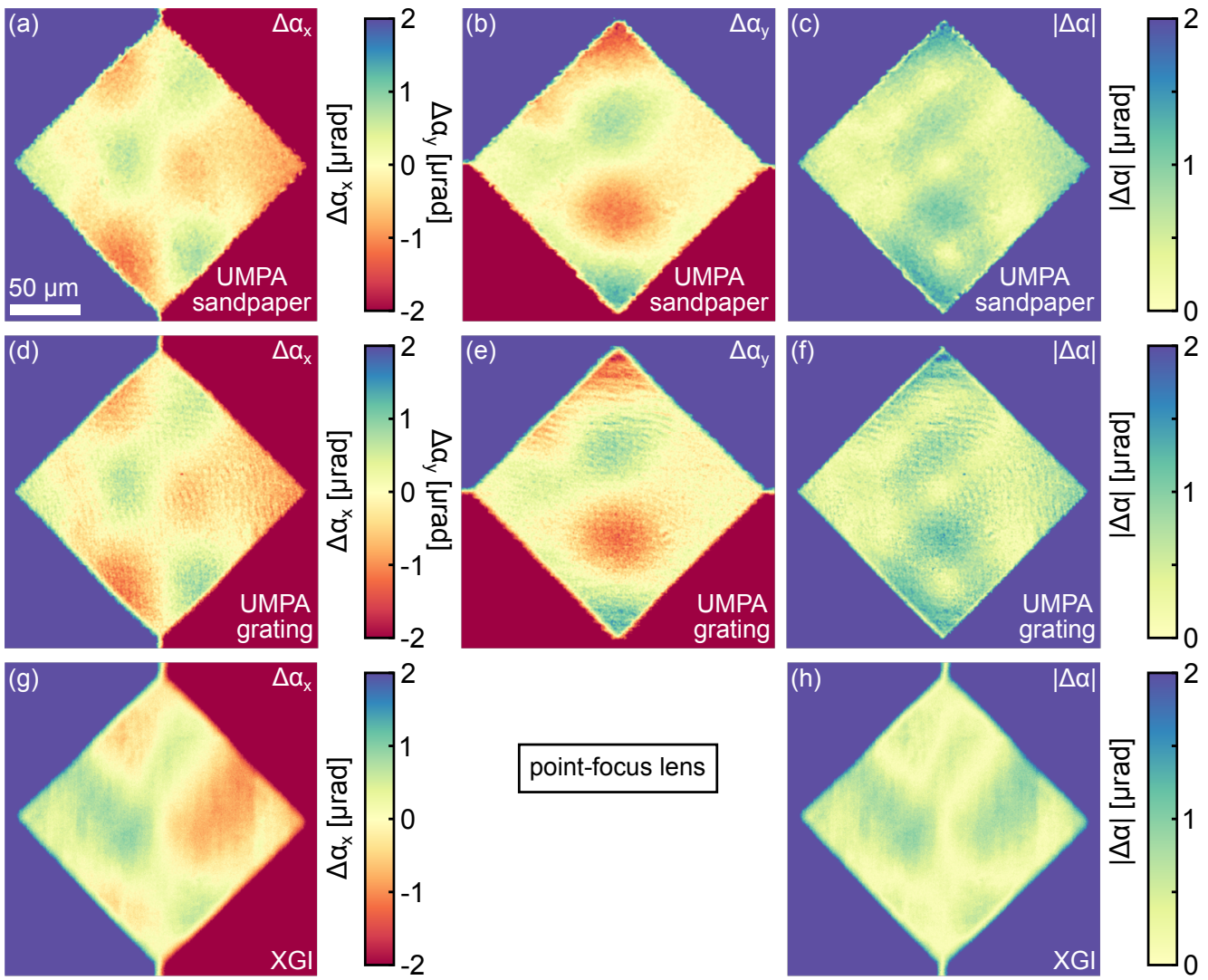

line-focus lens
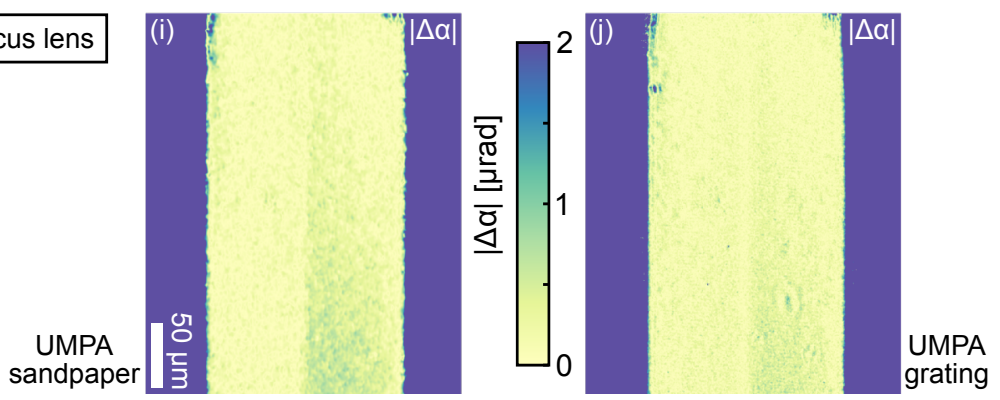

Fig. 4. Residuals from a linear fit to the refraction angle of lens B (wavefront gradient error) in (a), (d) the horizontal, (b), (e) the vertical direction, and (c), (f) absolute deviation, measured with the UMPA sandpaper and grating setups, respectively. Residuals from (g) the horizontal refraction angle and (h) absolute deviation in the horizontal, obtained from the two-grating interferometer. (i), (j) Absolute deviation from the horizontal refraction angle signal of lens A for the UMPA sandpaper and grating setups, respectively. 
from the horizontal and vertical signals. For the XGI case the absolute of the deviation in the horizontal is shown, as the setup is only sensitive in one direction.

The small grain-like artefacts visible in Figs. 4(a)-4(f) are a result of remaining features of the reference pattern in the reconstructed refraction signals. This is caused by the demagnification effect of the lens on the interference pattern, which is here neglected, but could in the future be included in the analysis for further improvement of the technique.

Large discrepancies from the expected focussing behaviour of lens B can be observed in both directions. Thanks to the two-dimensional sensitivity of the UMPA approach, the change in the lens properties can be fully characterised from a single data set. The deviations, which reach up to $3.0 \mu \mathrm{rad}$, are more pronounced in the vertical direction and largest in the areas around the centre of the aperture, indicating an alteration of the refractive index and/or thickness of the lens material in this region. This significant divergence within the lens aperture would result in strong changes of the desired focussing behaviour making the lens useless for further applications as an optical element.

The absolute deviation is also shown for lens A in Figs. 4(i) and 4(j) for the UMPA sandpaper and grating setups, respectively. The linear fit to the refraction angle was performed in the upper part of the lens. It can clearly be seen that the divergence from the expected refraction is significantly smaller than for the damaged lens. However, in the lower part deviations can be noted, which strongly increase when moving closer to the substrate. This has been observed previously for refractive lenses made from SU-8 material and has been attributed to shape errors of the lens due to tilted side walls and thermal stress during the manufacturing process $[25,58]$. These effects are more pronounced close to the substrate, where as a consequence the refraction behaviour cannot be modelled as a linear curve anymore, which leads to significant deviations of the measured refraction angle data from a linear fit. It has been shown that the shape errors result in a decrease of the measured focal length from the top to the bottom of the lens $[25,58,59]$. This effect can clearly be observed for lens A. Figure 5 illustrates the variation of the focal length over the height of the lens. The focal length $f$ was determined by performing a linear fit of the refraction angle for each row of the image and applying Eq. (6) to obtain $f$. As seen from Fig. 5, the focal length clearly decreases from the top to the bottom of the lens.

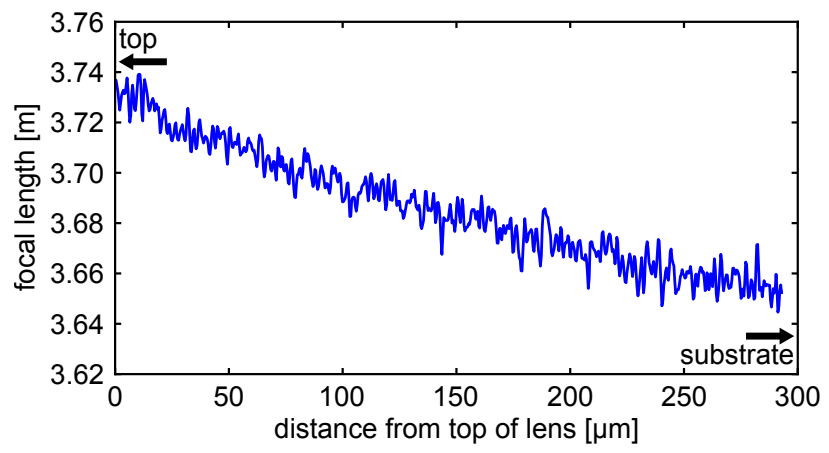

Fig. 5. Variation of the measured focal length along the height of lens A (line-focus lens). The decrease of the focal length closer to the substrate is due to shape errors more pronounced in this area.

\subsubsection{Influence of the deviations on the focal spot}

The imperfections in the wavefront created by the lenses caused by beam damage or shape errors led to deviations from the desired point or line focus. To visualise this, the measured wavefront was propagated along the beam direction $z$ starting from the detector plane using an angular spectrum approach [60], in which the Fourier transform of the wave field is multiplied with the 
transfer function:

$$
\mathcal{H}=\exp \left(i k z \sqrt{1-q^{2} \lambda^{2}}\right),
$$

where $z$ is the propagation direction of the wavefront, $\lambda$ the wavelength, $k=2 \pi / \lambda$ the wave vector and $q^{2}$ the sum of the squared spatial frequencies in $x$ and $y$, transverse to the propagation direction. Subsequently, an inverse Fourier transform is applied to the product.

Figure 6 shows cuts of the propagated wavefronts, obtained from the UMPA setup using
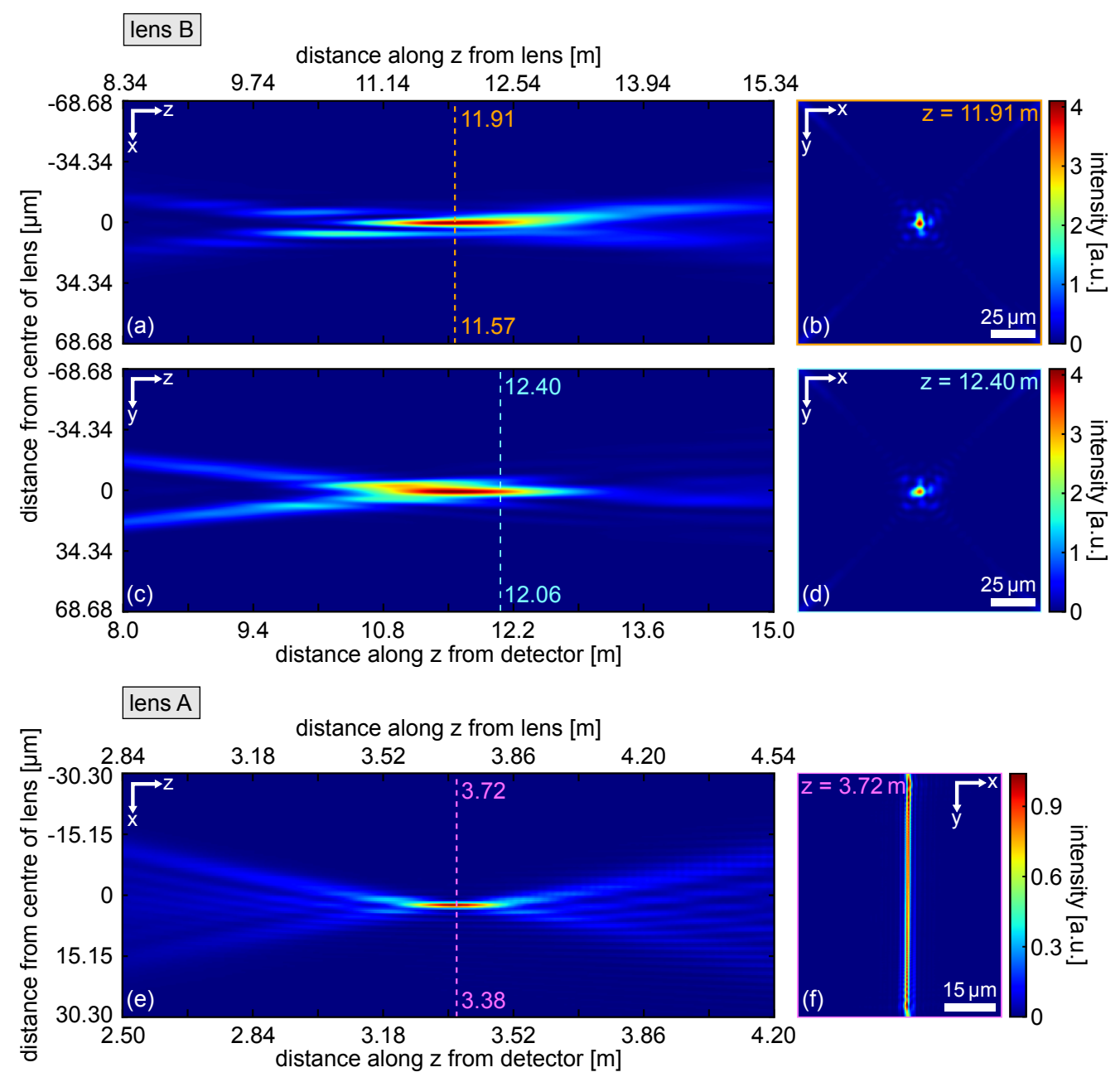

Fig. 6. Cuts through the propagated wave fields after the point-focus lens B (beam damage) and line-focus lens A (shape errors), calculated with an angular spectrum propagator from the phase signal measured with the UMPA grating setup in the detector plane. (a), (c) Longitudinal cuts through the centre of lens B in the $x z$ - and $y z$-planes, respectively. The dashed lines indicate the distance from the detector where the focal length was found for the horizontal (orange line) and the vertical (cyan line) focussing direction (numbers give the distance from the detector (bottom) and from the lens (top)). (b), (d) Transverse slices along the vertical $y$ through the wave field of lens B at the focal distances in the horizontal and vertical directions, respectively. (e) Longitudinal cut through the propagated wave field of lens $\mathrm{A}$ in the top part of the lens (pixel row 39 from the top) and distance of the focal line from the detector (magenta line) and (f) corresponding transverse slice at this z-position. 
the rotating grating, through the centre of the lens position. For lens B an asymmetry of the longitudinal cuts in the horizontal and vertical directions in Figs. 6(a) and 6(c), respectively, can be observed. Theoretically a single focal length of $f_{\mathrm{B}}=12.24 \mathrm{~m}$ would be expected in both focussing directions for the point-focussing lens B according to Eq. (7). The beam damage, however, gave rise to astigmatism of the lens, i.e. the focal planes in the two directions are located at different distances. The minimum beam size was found at $11.91 \mathrm{~m}$ (orange line) downstream of the lens, i.e. $11.57 \mathrm{~m}$ from the detector, for the horizontal and at $12.40 \mathrm{~m}$ (cyan line) from the lens, i.e. $12.06 \mathrm{~m}$ from the detector, for the vertical focussing direction. The transverse beam profiles at these distances are shown in Figs. 6(b) and 6(d). Furthermore, the diameter of the focal spot differs for the horizontal and vertical directions as visible in the transverse cuts in Figs. 6(b) and 6(d). Some spurious features can be observed in these images around the area of highest intensity, which are caused by the astigmatism. The size of the focal spot was estimated separately in the horizontal and the vertical directions by fitting a Gaussian curve to the line profile of the propagated wave field through the centre of the lens in the respective focussing direction, while neglecting the spurious features around the spot. The full width at half maximum of the fitted Gaussian can be seen as an estimate for the spot size. It is $4.7 \mu \mathrm{m}$ for the horizontal and $5.4 \mu \mathrm{m}$ for the vertical direction.

The propagation of the wave field of lens $\mathrm{A}$ is shown as a longitudinal cut in the $x z$-plane through the top part of the lens in Fig. 6(e). As discussed in the previous section, the $z$-position of the focal plane decreases over the height of the lens due to shape errors. It is located at $3.72 \mathrm{~m}$ downstream of the lens ( $3.38 \mathrm{~m}$ from the detector) for the upper part of the lens as indicated by the magenta line in Fig. 6(e). The transverse cut through the propagated wave field at this distance can be found in Fig. 6(f), which shows a sharp focus at the top of the lens. The change of the position of the focal line for different heights of the lens is illustrated in more detail by the propagated wave field cuts through different heights of the lens in Fig. 7 in Sec. 6.1 of the appendix. The nominal focal length of the lens calculated with Eq. (7) is given by $f_{\mathrm{A}}=3.74 \mathrm{~m}$. The width of the focal line, estimated by fitting of a Gaussian line as above, is approximately $1.3 \mu \mathrm{m}$.

\section{Conclusions and outlook}

We have demonstrated that the recently proposed UMPA method is suitable for X-ray wavefront sensing and optics characterisation. Beam damage and shape errors of two polymer X-ray refractive lenses were successfully detected by analysing the wavefront downstream of the lens qualitatively as well as quantitatively. We have furthermore shown the excellent agreement of the results obtained from an UMPA setup with a periodic and a random reference interference pattern and have validated them with conventional X-ray grating interferometry measurements. Combined with the flexibility and ease of implementation, this illustrates that UMPA is readily adaptable to many existing X-ray phase-sensitive setups and we expect it to find widespread applications for wavefront analysis and optics characterisation. As UMPA is a robust technique that does not require a high degree of spatial and lateral coherence and does not impose stringent restrictions on the setup length or alignment, it is suitable for the investigation of various kinds of optics and could in the future be transferred to laboratory sources without major efforts. This makes UMPA a perfect candidate for cost-effective routine inspection and quality testing of optics such as refractive lenses, allowing near at-wavefront characterisation without the need for costly and limited synchrotron access. This will be an important step for the improvement and development of optics fabrication. 


\section{Appendix}

\subsection{Focussing properties of the line focus lens $A$}

As observed above, the line focus lens A shows some shape errors caused by the manufacturing process that lead to a decrease of the focal length from the top to the bottom part of the lens. The effect on the focussing behaviour of the lens can be visualised by propagating the wavefront measured in the detector plane in a simulation using an angular spectrum propagator (see Eq. (8)). Figure 7 shows longitudinal and transverse cuts through the propagated wave field of lens A for different heights of the lens and different propagation distances. It can be seen from the longitudinal sections through the top, middle and bottom (close to the substrate) of the lens in Figs. 7(a), 7(c), and 7(e), respectively, that the position of the focal line changes over the height of the lens. The magenta dashed lines indicate the distance of the focal line from the detector for each height, which decreases closer to the substrate. Figures 7(b), 7(d), and 7(f) show the transverse cuts of the wave field at the corresponding focal lengths.

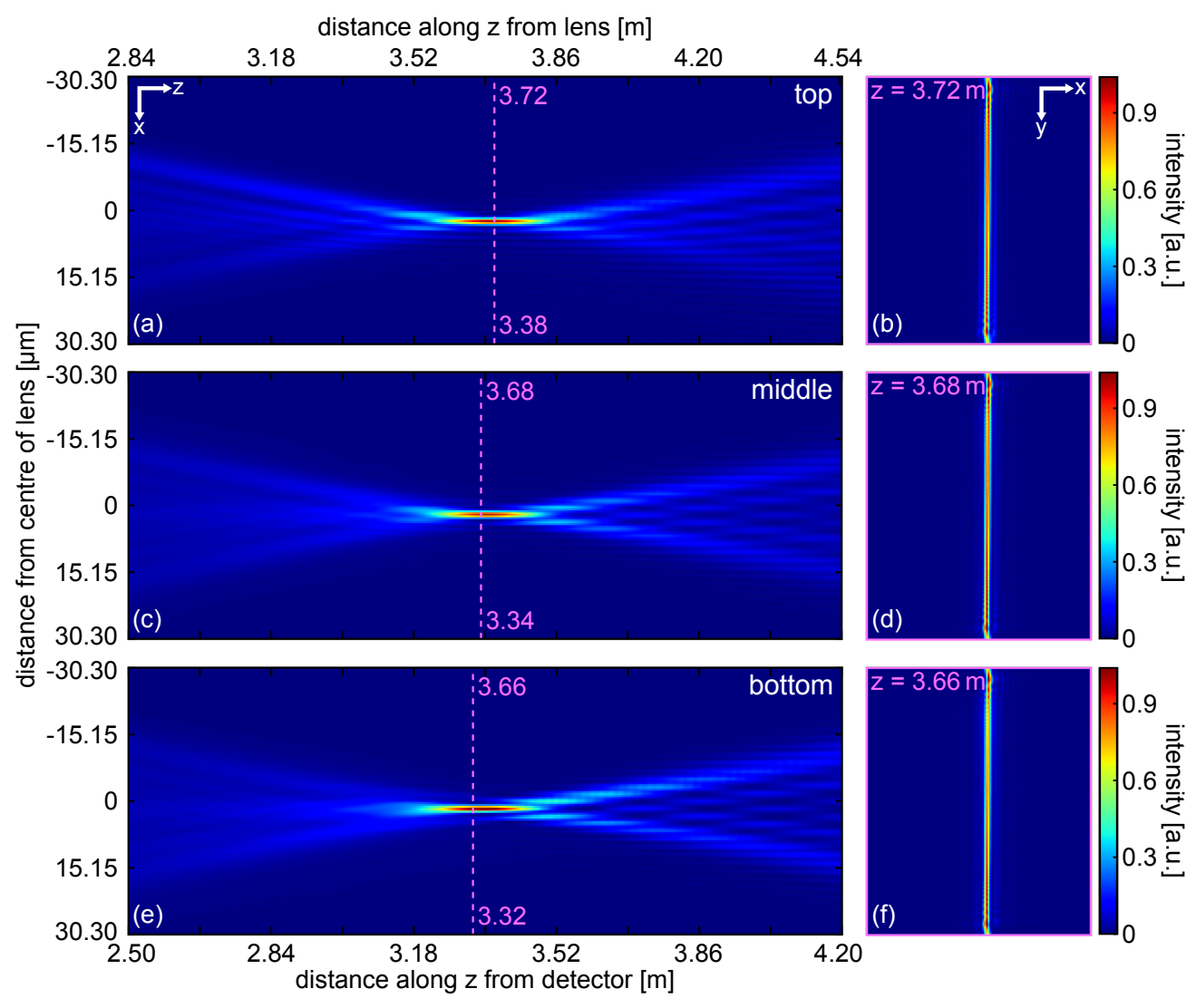

Fig. 7. (a), (c), (e) Longitudinal cuts ( $x z$-plane) through the propagated wave fields of lens A in the top, middle and bottom part of the lens. Magenta lines indicating the position of the calculated focal length measured from the detector plane (bottom value) or lens position (top value). (b), (d), (f) Transverse cuts at the focal distances at $z=3.72 \mathrm{~m}, z=3.68 \mathrm{~m}$, and $z=3.66 \mathrm{~m}$ from the lens. 


\subsection{Information about the $X$-ray exposure of the damaged lens $B$}

Beam damage occurred in the point-focussing SU-8 refractive lens (lens B) due to intense $\mathrm{X}$-ray radiation over a prolonged period of time prior to the characterisation measurements presented here. It was used at beamline BL10XU at SPring-8 synchrotron as part of a CRL array for beam focussing at an energy of $30 \mathrm{keV}$. Pre-focussing of the X-ray beam upstream of the lens under study was performed with a $2 \mathrm{~mm}$-diameter beryllium CRL resulting in a beam diameter of $150 \mu \mathrm{m}$. The beam was then cut down using a pair of slits, so that an X-ray beam of $100 \times 100 \mu \mathrm{m}^{2}$ effectively impinged onto the SU-8 lens. The photon flux on the investigated lens was $10^{13}$ photons $/\left(\mathrm{s} \cdot \mathrm{mm}^{2}\right)$. The CRL remained in the X-ray beam for 523 days with a daily X-ray irradiation of approximately 4 hours, summing up to about 2100 hours of exposure. The total dose absorbed by the lens during this time was approximately $27.3 \mathrm{~kJ} / \mathrm{mm}^{3}$. This accumulated dose led to a change in the properties of the lens material and hence the refraction behaviour, resulting in deviations of the wavefront from a parabolic shape, broadening of the focal spot and astigmatism, as investigated in the previous sections.

\section{Funding}

European Research Council (ERC) FP7 (StG 279753).

\section{Acknowledgments}

We acknowledge Diamond Light Source for time on the the I13-1 Coherence beamline under experiment number MT14562-1. We acknowledge Kaz Wanelik, Simon Logan, Xiaowen Shi, Ulrich Wagner and Andrew Wilson from Diamond Light Source for their support in the preparation and setup of the experiment. 